\title{
G scome \\ Identification of Key ncRNAs and ceRNA Network in DR via Bioinformation Analysis
}

\section{Jingru Li}

Department of Cardiology, The First Affiliated Hospital of Kunming Medical University, Kunming, Yunnan

\section{Chaozhong Li}

Department of Emergency, The First Affiliated Hospital of Kunming Medical University, Kunming,

Yunnan

\section{Xinyu Wu}

Department of Cardiology, The First Affiliated Hospital of Kunming Medical University, Kunming, Yunnan

\section{Shuai Yu}

Department of Cardiology, The First Affiliated Hospital of Kunming Medical University, Kunming, Yunnan

\section{Guihu Sun}

Department of Cardiology, The First Affiliated Hospital of Kunming Medical University, Kunming, Yunnan

\section{Peng Ding}

Department of Emergency, The First Affiliated Hospital of Kunming Medical University, Kunming,

Yunnan

Si Lu

Department of Cardiology, The First Affiliated Hospital of Kunming Medical University, Kunming, Yunnan

\section{Lijiao Zhang}

Department of Cardiology, The First Affiliated Hospital of Kunming Medical University, Kunming, Yunnan

\section{Ping Yang}

Department of Cardiology, The First Affiliated Hospital of Kunming Medical University, Kunming, Yunnan

\section{Yunzhu Peng}

Department of Cardiology, The First Affiliated Hospital of Kunming Medical University, Kunming, Yunnan Jingyun Fu

Department of Endocrinology, The First Affiliated Hospital of Kunming Medical University, Kunming, Yunnan

\section{Luqiao Wang ( $\nabla$ wlq8360@163.com )}

Department of Cardiology, The First Affiliated Hospital of Kunming Medical University, Kunming, Yunnan

\section{Research Article}

Keywords: Diabetic retinopathy, Biomarker, Microarray, Bioinformatics analysis, RNA regulatory pathways

Posted Date: February 22nd, 2022 
DOI: https://doi.org/10.21203/rs.3.rs-1291708/v1

License: (c) (1) This work is licensed under a Creative Commons Attribution 4.0 International License. Read Full License 


\section{Abstract}

This study aimed to find potential diagnostic biomarkers for diabetic retinopathy (DR) , explore its ceRNA mechanism and analyze the role of immune cell infiltration in DR.

\section{Method.}

Three microarray datasets for human retina tissues and aqueous humor in DR were downloaded from the Gene Expression Omnibus (GEO) database. First, differentially expressed miRNAs (DE-miRNAs) and differentially expressed mRNAs (DE-mRNAs) were identified by R software. Then xCell and ImmuCellAI were used to evaluate the infiltration of immune cells in DR retina tissues. BioGPS was used to identify tissue/organ-specific genes; DE-mRNA was enriched, and a protein-protein interaction (PPI) network was constructed to understand its functions and enrichment pathways and identify hub genes. Finally, we used target gene prediction software to construct competing endogenous RNA (ceRNA) networks for hub genes and visualized them with Cytoscape.

\section{Result.}

A total of 90 DE-miRNAs were identified in the GSE140959 datasets. Among these DE-miRNAs, the top 3 upregulated miRNAs, hsa-miR-3960, -548y and -7, may be potential biomarkers for diagnosis of DR. In the GSE160306 datasets, we recognized 147 DE-mRNAs. The xCell analysis identified immune cells that are significantly changed after DR, such as memory B-cells, eosinophils and M2 macrophages. Immune cell infiltration analysis found that natural killer T (NKT) cells, mucosal-associated invariant T (MAIT) cells, exhausted T cells and macrophages may be involved in the DR process. Comprehensive analysis showed that the negative regulation of cellular defense response and adaptive immune response was the main process of significant enrichment of DE-mRNAs. Among these DE-mRNAs, we identified 91 tissue/organspecific expressed genes and intersected with 10 cytoHubba genes to finally obtain three central hub genes: IL6, SLAMF1 and CCL8. And finally constructed PP12613/hsa-miR-2114-3p/IL6, LINC00960/hasmiR-6891-5p/SLAMF1 and KCNQ10T1/hsa-miR-7151-3p/CCL8 network with potential regulatory effect on DR progression.

\section{Conclusions.}

In conclusion, we believe that hsa-miR-3960, $-548 \mathrm{y}$ and -7 can be used as diagnostic markers for DR, and PP12613/hsa-miR-2114-3p/IL6, LINC00960/has-miR-6891-5p/SLAMF1 and KCNQ10T1/hsa-miR-7151$3 p / C C L 8$ regulatory networks play important roles in the occurrence and progression of DR. At the same time, the infiltration of immune cells is also an important part of DR pathology, regulating the severity of DR. Our data provide compelling insights into the pathogenesis and potential therapeutic targets of DR.

\section{Introduction}


Diabetic retinopathy (DR) is the most common microvascular complication of diabetes, and the risk of blindness in diabetic patients is 25 times that of non-diabetic patients ${ }^{1,2}$. Since the development of diabetes mellitus (DM) is progressive. It causes severe visual impairment, resulting in treatment difficulty if $D R$ is not promptly diagnosed and treated ${ }^{3}$. Therefore, how to diagnose $D R$ at an early stage and control its progression has become the focus of attention. ${ }^{4}$. The current diagnosis of DR is based on the clinical features observed on a comprehensive ophthalmic examination ${ }^{5}$. However, this method has several drawbacks: first, it is delayed; furthermore, it is not conducive to rapid diagnosis due to the high cost, time-consuming, and complexity of ophthalmic examinations. Therefore, it is particularly important to find biomarkers that are more specific for DR and can provide more rapid diagnosis for elucidating the molecular mechanism of DR occurrence and assisting DR treatment.

Intensive research into diabetes over the past 10 years has found that obesity-related inflammation in adipose tissue is one of the main causes of type 2 diabetes and is driven by different immune cells $s^{6,7,8,9}$. Accumulation of macrophages in the adipose tissue of diabetic patients alters the polarization of macrophages, resulting in an increase in M1 pro-inflammatory macrophages ${ }^{10}$. In addition, proinflammatory bone marrow-derived bone marrow cells, as well as T cells (cytotoxic CD8+, Th1 and Th17 pro-inflammatory cells) and NK cells, are recruited to adipose tissue, while normal Treg complement decreases, leading to an overall increase in adipose tissue inflammation in diabetes ${ }^{11}$. These are related to the pathogenesis of diabetic retinopathy. DR occurs in the context of chronic systemic inflammation. Circulating inflammatory factors can damage the function of the retina. Therefore, elucidating the complex mechanism of immune response will help to develop new therapies for the prevention and/or treatment of DR.

In recent years, more and more studies have shown that immune cell infiltration plays an important role in the occurrence and development of DR. For example, antigenicity associated cytotoxic $T$ lymphocytes selectively destroy pericytes and cause vascular collapse in diabetic retinopathy ${ }^{12}$. Intravitreal injection of anti-IL-17A monoclonal antibody can effectively improve Müller cell dysfunction, vascular leukocyte stagnation and vascular leakage in retina ${ }^{13}$. Therefore, from the perspective of the immune system, evaluating the infiltration of immune cells in the DR microenvironment and determining the differences in the composition of infiltrating immune cells are of great value for elucidating the molecular mechanism of DR and developing new immunotherapy targets. ImmuCellAl, a method based on gene set characteristics, accurately estimates the abundance of 24 immune cell types from gene expression data, including $18 \mathrm{~T}_{\text {cell subsets }}{ }^{14}$. At present, it has been widely used in the research of lung adenocarcinoma ${ }^{15,16}$, endometrial cancer ${ }^{17}$ and Kawasaki disease ${ }^{18}$. Currently, ImmuCellAI has not been used to analyze immune cell infiltration in DR. Therefore, in this paper we tried to combine ImmuCellAI with DR to analyze immune cell infiltration in DR. In addition, xCell is also used in this paper: This is a new algorithm that combines gene set enrichment and deconvolution method, aligns the enrichment fraction of each gene through the algorithm, and provides its gene characteristics in 64 immune cells, including adaptive immune cells and innate immune cells ${ }^{19}$. 
Currently, transcriptomic and microarray analyses have been widely used in several diseases, including

multiple tumors and DR, to identify novel biomarkers to assist in diagnosis and therapy ${ }^{20-24}$. In addition, competitive endogenous RNA (ceRNA) networks can elucidate new mechanisms for disease development in transcriptional regulatory networks ${ }^{25}$. Through the combination of microarray and bioinformatics analyses, it is possible to explore potential key genes and pathway networks that are closely related to the development of diseases.

Therefore, in this study, deep bioinformatics analysis was performed using the GEO database to screen out differentially expressed genes, and expression analysis was used to screen and identify diagnostic markers for DR. For the first time, ImmuCellAI was used to analyze the difference of immune infiltration between DR retinal tissue and normal tissue in 24 immune cell subsets for all retinal genes; $x$ Cell was used to analyze the enrichment fraction of retina-related genes in 64 immune cells. Subsequently, the functional enrichment of differential genes and protein-protein interaction (PPI) network are carried out, and examples of abnormal mRNA and ncRNA constituting ceRNA network are given to screen and determine diagnostic markers and clarify the mechanism of these gene interactions and synergistic driving DR development. Overall, this work provides insight into the mechanisms of DR disease development at the transcriptomic level and explores potential biomarkers for early diagnosis and treatment of DR. In addition, we studied the relationship between diagnostic markers and infiltrating immune cells to better understand the molecular immune mechanism during the development of DR.

\section{Materials And Methods}

\section{Expression Profile of miRNA and mRNA in Microarray Data From Patients With DR}

The three microarray datasets of patients with DR microarray datasets were collected from National Institutes of Health $(\mathrm{NIH})$-National Center for Biotechnology Information (NCBI) -Gene Expression Omnibus (GEO) databases (https:/www.ncbi.nlm.nih.gov/gds/). The detailed information of these GEO datasets was shown in Table 1. In the initial microarray experiments, different samples were used for different datasets: aqueous humor (dataset GSE140959) and retina (datasets GSE160306 and GSE160308). Therefore, we used GSE140959 to predict DR-related biomarkers, GSE160306 and GSE160308 datasets to construct ceRNA regulatory networks (since the GSE160306 and GSE160308 datasets used the same retinal samples, giving us the effect of comparing ceRNAs in the same cell types). Of note, our approach was well justified. For example, as a common practice, we $\mathrm{e}^{26,27}$ and others ${ }^{28}$ often studied gene expression in pathophysiological conditions. 
Table 1

Information of selected microaray datasets.

\begin{tabular}{|lllllll|}
\hline GEO accession & Species & Source tissue & Sample & Data & Attribute \\
\hline GSE140959 & Human & Aqueous humor & 4 & 6 & miRNA & Test set \\
\hline GSE160306 & Human & Retina & 20 & 59 & mRNA & Test set \\
\hline & & & & & IncRNA & Validation set \\
\hline GSE160308 & Human & Retina & 20 & 59 & miRNA & Validation set \\
\hline
\end{tabular}

Table 2

Distribution of tissue/organ-specific expressed mRNAs identified by BioGPS.

\begin{tabular}{|llc|}
\hline System/Organ & Genes & Counts \\
\hline Digestive system & $\begin{array}{l}\text { CCND1, FOSL1, C5, ACSL4, C8B, ASGR1, CPA6, CYP2E1, } \\
\text { HAAO, SDS, ANG, GNMT, TST, SAA4, ACOX2, ALDH1L1, } \\
\text { PXMP2, PROC, ACADS, GLS, CST1, GIPC2, TGM3, MUC2, }\end{array}$ & 28 \\
\hline ST6GALNAC1, PYY, SI, MTTP & \\
\hline Hematology/Immunology & $\begin{array}{l}\text { SLAMF1, CCR10, CCR6, IL4R, SP140, IGLL1, ANKRD27, GNLY, } \\
\text { CD244, PREX1, TBX21, NRP2, HEMGN, ICAM4, MAP2K3, } \\
\text { SLC22A4, SPTA1, FAXDC2, IFIT1, NAMPT, H2BC4, CCL8, IL6 }\end{array}$ & 23 \\
\hline Endocrine System & $\begin{array}{l}\text { GABRA2, PMCHL1, ARHGAP36, PRSS16, ID4, MPPED2, } \\
\text { Respiratory system }\end{array}$ & $\begin{array}{l}\text { HMGA2, LAMC2, GJB2, MICA, SFTPA2, CYP4B1, CLDN5, } \\
\text { MSLN }\end{array}$ \\
\hline Nervous system & $\begin{array}{l}\text { AVIL, PSG11, TCHH, SLC44A2, PSG9, RPL10L, SPATA4, } \\
\text { LEFT1 }\end{array}$ & 8 \\
\hline Urinary system & ATP1B3, ABCC3, SLC3A1, PART1, KLK2 & 5 \\
\hline $\begin{array}{l}\text { Circulatory/Cardiovascular } \\
\text { System }\end{array}$ & HAPLN1, CKMT2, NPPA, XIRP1, MYH6 & 5 \\
\hline Reproductive system & PLAC4, SI, RGR, C1QTNF5 & 4 \\
\hline Adipose tissue & FADS1 & 1 \\
\hline Eye & KCNV2 \\
\hline
\end{tabular}


Table 3

10 hub genes identified by five algorithms of cytoHubba.

\begin{tabular}{|llcll|}
\hline $\begin{array}{l}\text { Gene } \\
\text { symbol }\end{array}$ & Description & Log2FC & $\begin{array}{l}\text { P- } \\
\text { value }\end{array}$ & Regulation \\
\hline IL6 & Interleukin 6 & -1.12 & 0.019 & Downregulation \\
\hline CXCL9 & C-X-C motif chemokine ligand 9 & -4.27 & 0.005 & Downregulation \\
CCL8 & C-C motif chemokine ligand 8 & -2.01 & 0.041 & Downregulation \\
\hline CRYGC & Crystallin gamma C & -3.15 & 0.019 & Downregulation \\
CXCL11 & C-X-C motif chemokine ligand 11 & -2.52 & 0.014 & Downregulation \\
\hline SIGLEC1 & Sialic acid binding Ig like lectin 1 & -1.06 & $<$ & Downregulation \\
\hline FCGR2B & Fc gamma receptor lib & 1.15 & 0.014 & Upregulation \\
\hline SLAMF1 & Signaling lymphocytic activation molecule & -1.70 & 0.012 & Downregulation \\
\hline family member 1 & Complement C3d receptor 2 & -1.69 & $<$ & Downregulation \\
\hline ACAN & Aggrecan & 2.1 & 0.006 & Upregulation \\
\hline ImmUne and Stromal Cells analyses for the & Microarrays \\
\hline Datasets & & &
\end{tabular}

Recently published xCell (xCell, http://xCell.ucsf.edu/) is a new gene signature-based analysis method for inferring the characteristic values of genes in 64 immune and stromal cell species. It has been extensively analyzed in silico and compared to cellular immunophenotypes ${ }^{19}$. By applying xCell to the microarray data and the Wilcoxon method for variance, the estimated proportion of immune and stromal cell types can be obtained for each retina sample gene. The cut-off values for the cell analyses were $p<0.05$. Cell types were categorized into lymphoid, myeloid, stromal, stem cells and others. Violin plots are used to visualize the expression of different cell types in the xCell analysis results.

\section{Immune cell Infiltrate Analyses}

ImmuCellAI (ImmuCellAI, https://bioinfo.life.hust.edu.cn/web/ImmuCellAI/) ${ }^{14}$ is used to identify different immune cell types from RNA-seq results. Upload the gene expression matrix data to ImmuCellAl to obtain the immune cell infiltration matrix. Then cluster analysis of immune cell infiltration matrix data, and draw a two-dimensional PCA cluster diagram. The correlation heat map shows the correlation of genes in 24 types of immune cell infiltration, and the violin plot shows the difference in immune cell infiltration.

\section{Functional Enrichment Analyses}


The biological functions of identified DE-mRNAs of interest were assessed using the Metascape bioinformatics resources (Metascape, a gene annotation \& analysis resource, http://metascape.org, version 3.5 $)^{29}$. Briefly, shared DE-mRNAs were imported into Metascape, and GO and KEGG enrichment analyses were then conducted. For GO analyses, enriched biological processes (BPs), molecular functions (MFs), and cellular components (CCs) were assessed, and Kyoto Encyclopedia of Genes and Genomes (KEGG) pathway enrichment analyses.

\section{Protein-Protein Interaction Analysis}

Protein-protein interaction (PPI) networks were generated from the STRING database (STRING, https://string-db.org/). A score of 0.4 (medium confidence) was set as a threshold. Next, to access hub genes, the visualized regulatory network was constructed using the Cytoscape software (version 3.4.0, http://chianti.ucsd.edu/cytoscape-3.4.0/) ${ }^{30}$. Minimal Common Oncology Data Elements (MCODE) was used to identify significant gene clusters and obtain cluster scores (filter criteria: degree cut-off $=2$; node score cut-off $=0.2$; -core $=2$; max depth $=100$ ). CytoHubba was used to identify significant genes in this network as hub genes ${ }^{31}$. We used five algorithms, namely Degree, Maximal Clique Centrality (MCC), Maximum Neighborhood Component (MNC), Density of Maximum Neighborhood Component (DMNC), and Clustering Coefficient, to calculate the top 10 hub genes ${ }^{32,33}$

\section{Identification of tissue/organ-specific expressed genes}

To understand the tissue/organ-specific expression of these DEGs, the online tool BioGPS (BioGPS, http://biogps.org/) was used to analyze the tissue distribution ${ }^{34}$. The screening criteria were as follows: (1) transcripts that mapped to a single organ system with an expression value of $>10$ multiples of the median, and (2) second-most-abundant tissue's expression was no more than a third as high ${ }^{34}$. The genes obtained by these criteria were considered to be tissue-specific genes.

\section{Prediction of target miRNAs}

We used three online miRNA databases, namely, RNA22 (https://cm.jefferson.edu/rna22/) ${ }^{35}$, miRWalk (http://mirwalk.umm.uni-heidelberg.de/) ${ }^{36}$, and mirDIP (http://ophid.utoronto.ca/mirDIP/) ${ }^{37}$ to predict the target miRNA of the hub gene, screen out the common miRNAs in the three databases and intersect them with GSE160308-miRNA, and select the miRNA with the largest fold change in GSE160308 as the target miRNA. The messenger RNA (mRNA)-miRNA co-expressed network based on the relationship between mRNAs and miRNAs was constructed by using Cytoscape.

\section{Construction of ceRNA networks}

DIANA-LncBase ${ }^{38}$ (https://diana.e-ce.uth.gr/Incbasev3, version 3.0) and RNA22 ${ }^{35}$ (https://cm.jefferson.edu/rna22/Interactive/) were used to predict IncRNAs that interacted with the selected miRNAs ${ }^{39}$. The shared IncRNAs of DIANA and RNA22 were intersected with the GSE160306IncRNA database to obtain the intersection IncRNA. We selected the IncRNA with the largest fold change 
and entered by NCBI as the target. Then the CeRNA network based on the interaction between mRNA, miRNA and IncRNA was constructed using Cytoscape.

\section{Statistical Analysis of Microarray Data}

The two-tailed Students' t-test was used to identify the differences between groups. We used the $\mathrm{R}$ statistical analysis package (version 3.5.3) to statistically analyze the data. An alpha value of $P<0.05$ was measured and considered a statistically significant event or comparison in this study.

\section{Ethics approval and consent to participate}

The NCBI-GEO database used in the article belongs to the public database. The patients involved in the database have obtained ethical approval. Users can download relevant data for free to conduct research and publish relevant articles. Our research is based on open-source data, so there are no ethical concerns and other conflicts of interest.

\section{Results}

\section{Screening of Diagnostic Markers in Human Aqueous Humor of DR}

The GEO dataset (GSE140959) was included in our study. GSE140959 had 10 samples, including 6 aqueous humor samples from patients with diabetes mellitus (DM) Type II with DR (DMII-DR) and 4 aqueous humor samples from DM Type II with non-DR (DMII-NDR). As shown in the cluster heat maps and volcano plots in Fig. 1A and 1B, a total of 90 differentially expressed miRNAs (DE-miRNAs) (49 upregulated and 41 downregulated) were identified in the GSE140959 dataset. Among the upregulated miRNAs, the top 3 miRNAs in DR plasma were: hsa-miR-3960 ( $F C=1.84, p=0.0228$ ), hsa-miR-548y $(\mathrm{FC}=1.66, p=0.0099)$ and hsa-miR-7 ( $\mathrm{FC}=1.64, p=0.0284)$. Due to miRNA's good diagnostic performance in aqueous humor, we combined with the top 3 highest expression levels to identify better biomarkers. Compared with established DR aqueous humor, hsa-miR-3960, hsa-miR-548y and hsa-miR-7 were the top 3 up-regulated in DR with statistical significance (Fig. 1C). Therefore, we hypothesize that hsa-miR-3960, hsa-miR-548y and hsa-miR-7 may be biomarkers for early diagnosis of DR based on our present samples.

\section{Identification of DE-mRNAs in the Human Retina}

The GEO dataset (GSE160306) was included in our study. GSE160306 had 79 samples, including 59 retina tissues from patients with $D R$ and 20 retina tissues from people with non-DR. As shown in the cluster heat map and volcano plot in Fig. 2A and 2B, a total of 147 differentially expressed mRNAs (DEmRNAs, $p<0.05,|\log 2 \mathrm{FC}|>1$ ) (38 upregulated and 109 downregulated) were identified in the GSE160306 dataset.

\section{Immune Cell Infiltration Results}


PCA cluster analysis is a method to test the consistency of biological repetition and the differences between different groups. PCA cluster analysis results of immune cell infiltration showed that there was a significant difference in immune cell infiltration between the DR sample and the control sample (Fig. 3A). Correlation heat maps of 24 immune cells showed that there was a significant positive correlation between natural killer T (NKT) cells and exhausted T cells, and between mucosal-associated invariant $T$ (MAIT) cells and macrophages. There is a significant negative correlation between NKT cells and MAIT and macrophages; In addition, there is also a negative correlation between exhausted T cells and MAIT

cells and macrophages (Fig. 3B). The violin plot of the immune cell infiltration difference showed that, compared with the normal control sample, natural killer T cells and exhausted T cells and mucosalassociated invariant T cells infiltrated less, while macrophages infiltrated more (Fig. 3C).

\section{Immune and stromal cells deconvolution analyses}

In order to determine the cell types involved in the DR process, we used xCell to convert gene expression data into cell-type enrichment scores. The cell type results of the GSE160306 data set are shown in Fig. 4A-E. There are 5 significantly different immune cell types between the control group and the DR group, including: memory B-cells, eosinophils, lymphatic endothelial cells, plasmacytoid dendritic cells and macrophages M2. Of note, we then intersected the results of ImmucellAI with xCell and found that macrophages existed simultaneously in both methods of immune infiltration, more illustrating the important role of macrophages in Dr progression (Fig. 4F).

\section{Pathway Enrichment Analyses.}

The consequences of GO analysis indicated that these DE-mRNAs were associated with diverse biological processes, molecular functions and cellular components. The top 3 biological processes those DE-mRNAs were involved in were: cellular defense response, negative regulation of adaptive immune response based on somatic recombination of immune receptors built from immunoglobulin superfamily domains, and negative regulation of adaptive immune response; the top 3 significant cellular components related to those DE-mRNAs were: anchored component of membrane, external side of plasma membrane, and cluster of actin-based cell projections; the top 3 significant molecular functions in which the DE-mRNAs participated were: steroid hydroxylase activity, oxygen binding and chemokine activity (Fig. 5A). KEGG pathway analysis showed those DE-mRNAs to be significantly enriched in phagosome, amoebiasis and salmonella infection (Fig. 5B).

\section{PPI Network Analysis, MCODE Cluster Modules}

The interaction network between proteins coded by DEGs, which was comprised of 136 nodes and 65 edges, was constructed by STRING and visualized by Cytoscape (Fig. 6A). The MCODE plugin was used to identify gene cluster modules. In this network, we identified two modules, which are shown in Fig. 6B-C, according to the filter criteria. Cluster 1 had the highest cluster score (score: 5, 5 nodes and 10 edges), followed by cluster 2 (score: 3,3 nodes and 3 edges). 


\section{Identification of the Tissue/Organ-specific Expressed Genes and and cytoHubba Gene Identification}

A total of 91 tissue/organ-specific expressed genes were identified by BioGPS (Table 2, Fig. 7A). We observed that most of these DE-mRNAs were specifically expressed in the digestive system (28/91, $30.77 \%)$. The second organ-specific expressed system was the hematology/immunology, which included 23 genes $(23 / 91,25.27 \%)$. This was followed by endocrine system $(8 / 91,8.79 \%)$, respiratory system (8/91, 8.79\%), nervous system (8/91, 8.79\%), urinary system $(5 / 91,5.49 \%)$, circulatory/cardiovascular system $(5 / 91,5.49 \%)$, reproductive system $(4 / 91,4.40 \%)$, adipose tissue $(1 / 91,1.09 \%)$ and eye $(1 / 91$, $1.09 \%)$. BioGPS results showed that the number of haematologic/immune system-localized DE-mRNA ranked second in 91 tissue/organ-specific expression results, demonstrating the critical role of the haematologic/immune system in DR (Fig. 7A). Next, we used the cytoHubba plugin to identify hub genes. The top 10 hub genes (Fig. 7B) were identified by intersecting the results from the five algorithms of cytoHubba including Degree, MCC, MNC, DMNC, and Clustering Coefficient ${ }^{40}$. These hub genes with detailed information are shown in Table 3. These genes are the most important in the PPI network and may play an important role in the pathogenesis of DR. As the most common autoimmune disease, a better understanding of immune-related mechanisms in DR is an important part of current research. The discovery of genes specifically expressed by the immune system in DR retina may contribute to the discovery of key targets in the pathogenesis of DR. Therefore, we intersected 10 hub genes of cytoHubba and 91 genes specifically expressed in the immune system (Fig. 7C). Ultimately, we obtained 3 immune system-specific expressed hub genes-IL6 ( $F C=0.46, p=0.0189)$, SLAMF1 ( $F C=0.3, P=0.0122)$ and CCL8 $(F C=0.25, p=0.0412)$ which were downregulated in DR retina (Fig. 7D).

\section{Prediction of Target miRNAs and Construction of the Co-expressed Network}

We used three online miRNA databases, RNA22, miRWalk and miRDIP, to predict target miRNAs of IL6, SLAMF1 and CCL8. Finally, we obtained 56 shared target miRNAs of IL6 (Fig. 8A), 606 shared target miRNAs of SLAMF1 (Fig. 9A), 67 shared target miRNAs of CCL8 (Fig. 10A). Next, we crossed the consensus miRNAs in the three databases with the 209 differentially expressed genes in the GSE160308 (included 59 retina tissues from patients with DR and 20 retina tissues from DM with non-DR) dataset by manual inspection and identified co-expressed miRNAs with the same expression trend. That is: if the expression of the mRNA is down-regulated (lower in the retina in DR patients compared to non-DR), we consider the predicted miRNA to be up-regulated in DR patients. Ultimately, we obtained that 8 out of 56 shared miRNAs were verified by the GSE160308 dataset of IL6, including 7 up-regulation and 1 downregulation in DR retina (Fig. 8B); 49 out of 606 shared miRNAs were verified by the GSE160308 dataset of SLAMF1, including 18 up-regulation and 31 downregulation in DR retina (Fig. 9B); 4 out of 67 shared miRNAs were verified by the GSE160308 dataset of CCL8, including 2 upregulation and 2 downregulation in DR retina (Fig. 10B). According to the miRNA-mRNA pairs hypothesis, we performed these verified miRNA to select the top 1 upregulated miRNAs in the retina of patients with DR for further analysis. Among the upregulated target miRNAs, the top changed miRNAs respectively were hsa-miR2114-3p (Fig. 8C), has-miR-6891-5p (Fig. 9C) and hsa-miR-7151-3p (Fig. 10C). Thus, the co-expressed 
network of IL6, SLAMF1 and CCL8 and target miRNAs were constructed: hsa-miR-2114-3p/IL6, has-miR6891-5p/ SLAMF1 and hsa-miR-7151-3p/CCL8.

\section{Construction of IncRNA-miRNA-mRNA Regulatory Network}

miRNAs is well known to induce gene silencing and downregulate gene expression by binding mRNAs. However, its upstream molecules, IncRNAs, can affect the function of miRNA by combining miRNA response elements, thus upregulating gene expression. This interaction between RNAs is called a ceRNA network ${ }^{25}$. Next, we used the online database, DIANA and RNA22 to predict the IncRNAs that interact with hsa-miR-2114-3p, has-miR-6891-5p and hsa-miR-7151-3p. We obtained 36 shared target IncRNAs of hsamiR-2114-3p (Fig. 8D-E), 120 shared target IncRNAs of has-miR-6891-5p (Fig. 9D-E) and 81 shared target IncRNAs of hsa-miR-7151-3p (Fig. 10D-E). Then, we performed the GSE160306, which included 59 retina tissues from patients with DR and 20 retina tissues from DM with non-DR, to verify shared target IncRNAs. Ultimately, we obtained the GSE160306 dataset to validate 3 of the 36 shared IncRNAs of hsamiR-2114-3p, including 1 up-regulated and 2 down-regulated IncRNAs in DR retina; 14 of the 120 shared IncRNAs of has-miR-6891-5p, including 4 up-regulated and 10 down-regulated IncRNAs in DR retina, and 3 of the 81 shared IncRNAs of hsa-miR-7151-3p, including 3 down-regulated IncRNAs in DR retina. According to the IncRNA-miRNA-mRNA pairs hypothesis, we performed these verified IncRNAs to select the downregulated IncRNAs in the retina of patients with DR for further analysis. Among the downregulated IncRNAs, we selected the effective IncRNAs with the largest fold change and entered by NCBI to construct a ceRNA network: IncRNA PP12613 (Fig. 8F), LINC00960 (Fig. 9F) and KCNQ10T1 (Fig. 10F). Thus, the ceRNA network was constructed: IncRNA PP12613/hsa-miR-2114-3p/IL6 (Fig. 8G), LINC00960/has-miR-6891-5p/SLAMF1 (Fig. 9G) and KCNQ10T1/hsa-miR-7151-3p/CCL8 (Fig. 10G). Hence, we propose the following IncRNA-miRNA-mRNA pathway: IncRNA PP12613/ hsa-miR-21143p/IL6, IncRNA LINC00960/ has-miR-6891-5p/SLAMF1 and IncRNA KCNQ10T1/ hsa-miR-7151-3p/CCL8; They might be a key regulatory pathway in the pathogenesis of DR.

\section{Discussion}

As the most common microvascular complication of diabetes, patients with DR have a 25 -fold higher risk of blindness than those without diabetes ${ }^{41}$. Due to the lack of early diagnostic indicators, patients with DR often lose the best opportunity for treatment, resulting in a poor prognosis. Therefore, finding specific diagnostic markers have profound significance for improving the prognosis of DR patients. With the rapid development of science and technology, bioinformatics has provided a powerful strategy for screening biofluids such as aqueous humor, vitreous humor, tear, and plasma.

In this study, we sought to identify diagnostic markers in aqueous humor for DR. We identified 49 upregulated miRNAs in the GSE140959 dataset, by comparing miRNAs expressed in human aqueous humor samples. Among the upregulated miRNAs, the top 3 miRNAs in DR plasma were: hsa-miR-3960 $(\mathrm{FC}=1.84, p=0.0228)$, hsa-miR-548y ( $\mathrm{FC}=1.66, p=0.0099)$ and hsa-miR-7 ( $\mathrm{FC}=1.64, p=0.0284)$. Due to the good diagnostic performance of miRNAs and the easy availability of aqueous humor, we defined the top 
3 miRNAs with the highest expression levels as biomarkers for DR diagnosis. Ding et.al' study showed that hsa-miR-3960 was significantly increased in the serum of type 2 diabetes mellitus (T2DM) patients compared with healthy people, and supposed serum hsa-miR-3960 is a highly accurate miRNA candidate molecule that can distinguish T2DM from healthy people ${ }^{42}$. Similar to our result, our study found that hsa-miR-3960 was upregulated in the DR aqueous humor ${ }^{43}$. The differential expression of hsa-miR3960 in aqueous humor suggests that it may be involved in the pathogenesis of diabetic retinopathy. Research by Li et al. proposed that miR-548y, which is differentially expressed and down-regulated in multiple myeloma, can be used as a diagnostic biomarker, but there is currently no research on miR-548y in DR. The research results of Yang et al. showed that miR-7 can attenuate the apoptosis of retinal pigment epithelial cells induced by high glucose and enhance cell activity through HoxB3 and $\mathrm{PI} 3 \mathrm{~K} / \mathrm{AKT} / \mathrm{mTOR}$ Signaling Pathways ${ }^{44}$; The study by Cao et al. proposed that miR-7 can inhibit the proliferation of retinal capillary endothelial cell and retinal pericytes in mice ${ }^{45}$. These studies have shown that differential miRNAs are involved in the biological process of DR and affect the prognosis of DR. Therefore, we propose that hsa-miR-3960, hsa-miR-548y and hsa-miR-7, which are differentially expressed and up-regulated in the aqueous humor of DR patients, have the potential to be effective biomarkers for the diagnosis of DR.

In addition, abnormal expression of genes is often closely associated with a range of pathological conditions, including DR. However, the key driver genes involved in the onset and progression. In addition, abnormal expression of genes is often closely associated with a range of pathological conditions, including DR. However, the key driver genes involved in the onset and progression of this condition remain to be fully elucidated. In this study, we identified 38 upregulated and 109 downregulated DRrelated DE-mRNAs in the GSE160306 dataset. These genes were associated with cellular defense response, negative regulation of adaptive immune response based on somatic recombination of immune receptors built from immunoglobulin superfamily domains, and negative regulation of adaptive immune response.

The immune response, which is characterized by the activation of classical immune cells, is considered one of the most vital mechanisms of DR. We found that natural killer T cells, exhausted T cells and mucosa-associated invariant $T$ cells which were infiltration decreased, and macrophages with increased infiltration may be involved in the progression of DR. A previous study showed that NKT cells and CD $4^{+}$ NKT cells were decreased in vivo in DR model mice, and the vascular permeability of retinal tissue was significantly increased after the human intervention to deplete NKT cells; It suggested that the infiltration degree of NKT cells was positively correlated with the severity of $\mathrm{DR}^{46}$; Sonoda et al.'s findings showed that NKT knockout mice exhibited reduced chorionic neovascularization capacity ${ }^{47}$. MAIT, an important cell in autoimmune regulation, recognizes derivatives of bacterial riboflavin metabolites presented by MHC IB related protein 1 (MR1) molecules and is an important effector cell of mucosal immunity. Its involvement in the regulation of diseases including autoimmune encephalomyelitis, human multiple sclerosis ${ }^{48}$, chronic liver disease, ${ }^{49}$ and type 1 diabetes $^{50}$. Diabetes associated autoantibodies were positive (AAb children were positive for two or more biochemical autoantibodies (insulin autoantibodies 
[IAA], insulinoma-associated-2 antibodies [IA-2A], GAD antibodies [GADA] and/or zinc transporter 8 autoantibodies [ZnT8A] ${ }^{++}$) The specific weight of MAIT cells appears reduced in patients with newly transformed type 1 diabetes ${ }^{50}$; Microglia, a type of mononuclear macrophage, is considered to be the resident macrophage of retinal tissue. When cells monitor an inflammatory stimulus, a phenotypic switch of $\mathrm{M} 1$ and $\mathrm{M} 2$ will occur ${ }^{51}$. M1 exhibits a classical pro-inflammatory effect, promoting the expression of pro-inflammatory factors such as IL-1 $\beta$, IL-6, IL-8, TNF-a; M2 has an anti-inflammatory phenotype and promotes clearance of debris to maintain homeostasis ${ }^{52}$. In vivo experiments in diabetic model mice revealed that activated microglia were highly proliferative and migratory ${ }^{53}$; Diabetes also induces Müller neuroglia cells to secrete vascular endothelial growth factor (VEGF) and TNF-a, which further aggravates the progression of $\mathrm{DR}^{54}$, And the quantitative growth of microglia was time-dependent ${ }^{55}$; Recent studies have shown that oxidative stress drives the activation of M1 like microglia by activating the STAT1 signaling pathway and promotes the release of proinflammatory cytokines under hypoxic conditions $^{56}$; IL-11/IL-11Ra Signaling and M2 macrophage density are positively correlated with angiogenesis in PDR ${ }^{57}$; These all illustrate that natural killer $\mathrm{T}$ cells, mucosal associated invariant $\mathrm{T}$ cells, and macrophages play important roles in the immune regulation of DR and are important regulators of the transformation and progression of the disease. Also illustrated that immune infiltration plays a non negligible role in the course of DR.

To further explore the role of immune cell infiltration in DR, we performed a comprehensive evaluation of DR immune infiltration using xCell. Specifically, memory B-cells, eosinophils, lymphatic endothelial cells, plasmacytoid dendritic cells and macrophages M2 were shown to have significant differences in the retina of DR patients. Research on diabetes by Cappellari et al. proposed that patients with impaired proangiogenic granulocytes (PAGs) (composed of eosinophils ( $>80 \%)$ and neutrophils $(<20 \%)$ ), endothelial cells are stimulated by granulocytes to form tubules Severe damage, aggravating the angiogenesis defect of diabetes ${ }^{58}$; The study by Majji et al. suggested that eosinophils infiltrate in the retinal tissues of some Eales' disease patients ${ }^{59}$, suggesting that eosinophils may have a regulatory role in eye diseases; In the epiretinal fibrovascular membranes tissue of patients with proliferative diabetic retinopathy (PDR), the level of CD163 M2 macrophages is positively correlated with the level of VEGF, suggesting that $\mathrm{M} 2$ macrophages may be involved in the angiogenesis of PDR ${ }^{57}$; in addition, many studies have proved that M2 Macrophages play a role in promoting angiogenesis and fibrosis in $D^{60-}$ 62; We then intersected the results of ImmucellAI with xCell and found that macrophages existed simultaneously in both methods of immune infiltration, more illustrating the important role of macrophages in DR progression.

After the hub genes that were screened by the PPI network, we further identified the immune systemspecific expressed hub gene associated with DR: IL6, SLAMF1 and CCL8. Therefore, we aimed to construct an mRNA-miRNA co-expression network and a ceRNA network to clarify the pathogenesis of DR at the transcriptome level. CeRNA is a transcript that acts as a miRNA sponge, and regulates each other at the post-transcriptional level by competing with shared miRNA for binding ${ }^{63}$. 


\section{IncRNA PP12613/hsa-miR-2114-3p/IL6 axis}

IL- 6 is a pleiotropic cytokine that induces the differentiation of B cells into plasma cells and regulates the proliferation and activation of T cells ${ }^{64}$. In addition, IL-6, as a proinflammatory cytokine, is associated with many ocular inflammatory diseases such as macular degeneration ${ }^{65}$ and $D R^{66,67}$. Further research on hub gene found that: IL6 level in DR patients is higher than normal, and IL6 level in PDR patients is higher than NPDR, suggesting that IL6 levels are positively correlated with the severity of DR ${ }^{68}$; there is microglia in the retinal pigment epithelium of hyperglycemic mice The accumulation of cells, IL-6-treated microglia produce and secrete tumor necrosis factor (TNF)-a, which activates NF-kB and reduces the level of zonula occludens (ZO)-1 in retinal pigment epithelium (RPE) cells, to destroy the outer-blood retinal barrier in diabetic retinopathy ${ }^{69}$. The study of Wang et al. also proposed that the NDRG2/IL-6/STAT3 signaling pathway participates in and aggravates the retinal damage of diabetes ${ }^{70}$;

Then, target miRNAs and downstream IncRNAs of mRNA-IL6 were predicted and used to construct ceRNA networks. According to the miRNA-mRNA pairs hypothesis, we performed the GEO dataset (GSE160308) to select the upregulated miRNAs in the retina of patients with DR for further analysis. Among the upregulated target miRNAs of IL6, the top 1 changed miRNAs were in DR: hsa-miR-2114-3p. Wang's study found that mir-2114-3p was involved in constituting the Cerna network of osteosarcoma (OS) and regulated tumor cell invasion ${ }^{71}$; Regarding to non-small cell lung carcinoma (NSCLC), studies have found that miR-2114, the precursor of miR-2114-3p, was expressed at high levels in the circulating peripheral blood of patients with adenocarcinoma (AC) and squamous cell carcinoma (SCC), suggesting that miR2114 can be used for diagnosis and treatment classification of lung cancer and precise diagnosis ${ }^{72}$; However, current studies on mir-2114-3p in DR are lacking.

Furthermore, the target IncRNAs of these miRNAs were predicted. According to the ceRNA hypothesis, we performed the verified IncRNAs by GSE160306 dataset to select the downregulated IncRNAs in the retina of patients with DR for further analysis. Among the validated target IncRNAs of hsa-mir-2114-3p, the IncRNA with the largest fold change and recruited by NCBI database was selected as the target of miRNA, from which we screened out: IncRNA pp12613. pp12613- is an exon-containing IncRNA located on chromosome 4q27. Since pp12613 is an emerging IncRNA, there is no report on the related research of pp12613 in DR. Waiting for us to further explore its biological function.

\section{IncRNA LINC00960/has-miR-6891-5p/SLAMF1 axis}

SLAMF1 is a classic inflammatory molecule, which is involved in Brucella infection ${ }^{73}$, bone marrow cell migration ${ }^{74}$ and HIV infection during systemic inflammation ${ }^{75}$. Magnusson's study shows that SLAMF1 is elevated in autoimmune endocrine diseases ${ }^{76}$; and the mutation of SLAMF1 on 1q21 is even stronger. Inflammation, which is related to the high incidence of type 2 diabetes in Indians ${ }^{77}$; but there is no relevant research on the mechanism of $\mathrm{DR}$, and we need to further explore it. 
As with the IL6 target miRNA and IncRNA discovery approach, we screened for SLAMF1 targets: has-miR6891-5p and LINC00960.

Zhong's research shows that miR-6891-5p is highly expressed in coronary artery disease and its target genes are related to basic biological terms such as biological process, cellular component, and molecular function $^{78}$; In exosomes from human bone mesenchymal stem cells (hBMSCs), mir-6891-5p also presented differential expression ${ }^{79}$;

In idiopathic pulmonary fibrosis, LINC00960 regulates the proliferation of fibroblasts ${ }^{80}$; high-grade bladder cancer cell-derived exosomes LINC00960 promotes the malignancy of low-grade bladder cancer cells through $\beta$-catenin signaling, Notch signaling, and Smad2/3 signaling pathways Behavior and induce epithelial-mesenchymal transition (EMT) ${ }^{81}$.

\section{IncRNA KCNQ10T1/hsa-miR-7151-3p/CCL8}

Chemokines Ligand 8 (CCL8), also known as monocyte chemotactic protein 2 (MCP2), is a small cytokine belonging to the $\mathrm{CC}$ chemokine family, which attracts and activates monocytes, $T$ cells, natural killer cells, eosinophils and many other immune cells participate in the inflammatory response ${ }^{82}$. CCL8 in HLA-B27related uveitis can be used as a local inflammation biomarker ${ }^{83}$; study of Irak et al. proposed that the reduction of CCL8 levels may affect the local area of Crohn's disease patients through cellular and molecular interactions between epithelial, immune and inflammatory cells The development and progression of intestinal inflammation and tissue destruction ${ }^{84}$; research on CCL8 in DR suggests that blocking vascular endothelial growth factor receptor 1 can interfere with CCL8 expression and prevent inflammation in diabetic retinopathy ${ }^{85}$; downstream of retinal inflammation, EC Transduction of proangiogenesis and/or inflammatory cytokines, resulting in increased synthesis and release of chemokines such as CCL8 and CXCL10, thereby regulating white matter balance and other cellular events related to DR vascular inflammation ${ }^{86}$; these studies suggest that CCL8 is largely involved in the pathological process of DR. But more detailed regulation mechanisms still need to be further explored.

At present, there is a lack of research on hsa-mir-7151-3p. Bai's research shows that after mir-7151-5p is down regulated, its downstream Wnt7a and Wnt/ $\beta$-The catenin signaling pathway is activated to promote the proliferation of thyroid carcinoma cells ${ }^{87}$.

The study by Shao et al showed that KCNQ10T1 was able to sponge to bind mir-1470 and further regulate epidermal growth factor receptor mRNA, promoting human retinal endothelial cell proliferation ${ }^{88}$; KCNQ10T1 is activated in diabetic corneal endothelial keratopathy and regulates high glucose induced corneal endothelial pyroptosis via the miR-214/caspase-1 signaling pathway ${ }^{89}$; Furthermore, kcnq1 ot 1 is also involved in the regulation of podocyte pyroptosis in renal inflammatory diseases ${ }^{90}$.

These all indicate the important role of differential miRNAs and signaling pathways, including ceRNA networks, in disease regulation, including DR. Ultimately, we constructed a new working model (Fig. 11) 
and proposed differential miRNAs: hsa-miR-3960, hsa-miR-548y, hsa-miR-7 and predicted constructed ceRNA network: IncRNA PP12613/hsa-miR-2114-3p /IL6, LINC00960/has-miR-6891-5p/SLAMF1, KCNQ10T1/hsa-miR-7151-3p/CCL8 may be novel and potent regulatory molecules regulating DR progression. Further revealed the regulatory mechanism of DR and enriched the disease regulatory network of DR. Of course, in our study, the sample size for analysis and verification is relatively small. Therefore, future studies need to increase the sample size and conduct prospective cohort studies to further confirm our views.

\section{Declarations}

\section{Data availability}

The National Institutes of Health (NIH)-National Center for Biotechnology Information (NCBI)-Gene Expression Omnibus (GEO) databases (https:/www.ncbi.nlm.nih.gov/gds/), xCell (xCell, http://xCell.ucsf.edu/), ImmuCellAI (ImmuCellAl, https://bioinfo.life.hust.edu.cn/web/ImmuCellAI/), Metascape (Metascape, a gene annotation \& analysis resource, http://metascape.org, version 3.5), STRING database (STRING, https://string-db.org/), Cytoscape software (version 3.4.0, http://chianti.ucsd.edu/cytoscape -3.4.0/), BioGPS (BioGPS, http://biogps.org/), RNA22 (https://cm.jefferson.edu/rna22/), miRWalk (http://mirwalk.umm.uni-heidelberg .de/), mirDIP (http://ophid.utoronto.ca/mirDIP/) and DIANA-LncBase (https://diana.e-ce.uth.gr/Incbasev3, version 3.0) databases were used in the generation or analysis of this article. All data generated and analyzed in this study can be found in these databases.

\section{AUTHOR CONTRIBUTIONS}

All authors have made significant contributions to the design and conception of the study, as well as data acquisition, analysis and interpretation, and participated in the drafting or revision of the manuscript. For the final version to be published, all authors have made the final approval, and have reached an agreement with the journal to which the article is submitted, and are willing to be responsible for all aspects of the work to ensure the accuracy or completeness of any part of the article. All the authors are willing to cooperate in the investigation and resolution of related issues in the follow-up of the article.

\section{Competing Interests Statement}

The authors declare that the research was conducted in the absence of any commercial or financial relationships that could be construed as a potential conflict of interest.

\section{Fundings}

This study was supported by the National Natural Science Foundation of China (No.81860073; 81760074; 82160439); Special Foundation Projects of Joint Applied Basic Research of Yunnan Provincial Department of Science and Technology with Kunming Medical University (No.2019FE001(-138)); Yunnan Provincial Department of Science and Technology (No.202001AT070039); Yunnan Health Training 
Project of High Level Talents (No.H-2018032); 100 Young and Middle-aged Academic and Technical Backbones of Kunming Medical University(No.60118260106); Young Talents of Yunnan Thousand Talents Plan(No.RLQN20200002); Clinical Medcial Center for Cardiovascular and Cerebrovascular Disease of Yunnan Province(No.ZX2019-03-01)

\section{References}

1. Simo-Servat, O., Hernandez, C. \& Simo, R. Usefulness of the vitreous fluid analysis in the translational research of diabetic retinopathy. Mediators of inflammation 2012, 872978, doi:10.1155/2012/872978 (2012).

2. Loukovaara, S. et al. Quantitative Proteomics Analysis of Vitreous Humor from Diabetic Retinopathy Patients. J Proteome Res 14, 5131-5143, doi:10.1021/acs.jproteome.5b00900 (2015).

3. Yin, L., Zhang, D., Ren, Q., Su, X. \& Sun, Z. Prevalence and risk factors of diabetic retinopathy in diabetic patients: A community based cross-sectional study. Medicine 99, e19236, doi:10.1097/MD.0000000000019236 (2020).

4. Reichard, P. et al. Intensified conventional insulin treatment retards the microvascular complications of insulin-dependent diabetes mellitus (IDDM): the Stockholm Diabetes Intervention Study (SDIS) after 5 years. J Intern Med 230, 101-108, doi:10.1111/j.1365-2796.1991.tb00415.x (1991).

5. Solomon, S. D. et al. Diabetic Retinopathy: A Position Statement by the American Diabetes Association. Diabetes Care 40, 412-418, doi:10.2337/dc16-2641 (2017).

6. Cinkajzlová, A., Mráz, M. \& Haluzík, M. Adipose tissue immune cells in obesity, type 2 diabetes mellitus and cardiovascular diseases. The Journal of endocrinology 252, R1-r22, doi:10.1530/joe-210159 (2021).

7. Deng, T. et al. Class II major histocompatibility complex plays an essential role in obesity-induced adipose inflammation. Cell Metab 17, 411-422, doi:10.1016/j.cmet.2013.02.009 (2013).

8. Anderson, E. K., Gutierrez, D. A. \& Hasty, A. H. Adipose tissue recruitment of leukocytes. Current opinion in lipidology 21, 172-177, doi:10.1097/MOL.0b013e3283393867 (2010).

9. Hevener, A. L. et al. Macrophage PPAR gamma is required for normal skeletal muscle and hepatic insulin sensitivity and full antidiabetic effects of thiazolidinediones. J Clin Invest 117, 1658-1669, doi:10.1172/jci31561 (2007).

10. Prieur, X. et al. Differential lipid partitioning between adipocytes and tissue macrophages modulates macrophage lipotoxicity and M2/M1 polarization in obese mice. Diabetes 60, 797-809, doi:10.2337/db10-0705 (2011).

11. Lui, P. P., Cho, I. \& Ali, N. Tissue regulatory T cells. Immunology 161, 4-17, doi:10.1111/imm.13208 (2020).

12. Adams, D. D. Autoimmune destruction of pericytes as the cause of diabetic retinopathy. Clinical ophthalmology (Auckland, N.Z.) 2, 295-298, doi:10.2147/opth.s2629 (2008). 
13. Qiu, A. W., Liu, Q. H. \& Wang, J. L. Blocking IL-17A Alleviates Diabetic Retinopathy in Rodents. Cellular physiology and biochemistry: international journal of experimental cellular physiology, biochemistry, and pharmacology 41, 960-972, doi:10.1159/000460514 (2017).

14. Miao, Y. R. et al. ImmuCellAl: A Unique Method for Comprehensive T-Cell Subsets Abundance Prediction and its Application in Cancer Immunotherapy. Advanced science (Weinheim, BadenWurttemberg, Germany) 7, 1902880, doi:10.1002/advs.201902880 (2020).

15. Sun, S. et al. Development and validation of an immune-related prognostic signature in lung adenocarcinoma. Cancer Med 9, 5960-5975, doi:10.1002/cam4.3240 (2020).

16. Zhao, C. et al. NEIL3 may act as a potential prognostic biomarker for lung adenocarcinoma. Cancer cell international 21, 228, doi:10.1186/s12935-021-01938-4 (2021).

17. Liu, J., Wang, Y., Mei, J., Nie, S. \& Zhang, Y. Identification of a Novel Immune Landscape Signature for Predicting Prognosis and Response of Endometrial Carcinoma to Immunotherapy and Chemotherapy. Front Cell Dev Biol 9, 671736, doi:10.3389/fcell.2021.671736 (2021).

18. Xie, Z. et al. Atlas of circulating immune cells in Kawasaki disease. Int Immunopharmacol 102, 108396, doi:10.1016/j.intimp.2021.108396 (2022).

19. Aran, D., Hu, Z. \& Butte, A. J. xCell: digitally portraying the tissue cellular heterogeneity landscape. Genome biology 18, 220, doi:10.1186/s13059-017-1349-1 (2017).

20. Carr, H. L., Turner, J. D., Major, T., Scheel-Toellner, D. \& Filer, A. New Developments in Transcriptomic Analysis of Synovial Tissue. Front Med (Lausanne) 7, 21, doi:10.3389/fmed.2020.00021 (2020).

21. Demircioglu, D. et al. A Pan-cancer Transcriptome Analysis Reveals Pervasive Regulation through Alternative Promoters. Cel/ 178, 1465-1477 e1417, doi:10.1016/j.cell.2019.08.018 (2019).

22. Youngblood, H., Robinson, R., Sharma, A. \& Sharma, S. Proteomic Biomarkers of Retinal Inflammation in Diabetic Retinopathy. International journal of molecular sciences 20, doi:10.3390/ijms20194755 (2019).

23. Gu, C. et al. Comprehensive analysis of angiogenesis-related genes and pathways in early diabetic retinopathy. BMC medical genomics 13, 142, doi:10.1186/s12920-020-00799-6 (2020).

24. Sun, H., Cheng, Y., Yan, Z., Liu, X. \& Zhang, J. Mining the proliferative diabetic retinopathy-associated genes and pathways by integrated bioinformatic analysis. Int Ophthalmol 40, 269-279, doi:10.1007/s10792-019-01158-w (2020).

25. Salmena, L., Poliseno, L., Tay, Y., Kats, L. \& Pandolfi, P. P. A ceRNA hypothesis: the Rosetta Stone of a hidden RNA language? Cell 146, 353-358, doi:10.1016/j.cell.2011.07.014 (2011).

26. Wang, L. et al. A comprehensive data mining study shows that most nuclear receptors act as newly proposed homeostasis-associated molecular pattern receptors. J Hematol Oncol 10, 168, doi:10.1186/s13045-017-0526-8 (2017).

27. Wang, L. et al. Novel extracellular and nuclear caspase-1 and inflammasomes propagate inflammation and regulate gene expression: a comprehensive database mining study. J Hematol Oncol 9, 122, doi:10.1186/s13045-016-0351-5 (2016). 
28. Li, Y. et al. A Functional Genomics Approach to Understand Variation in Cytokine Production in Humans. Cel/ 167, 1099-1110 e1014, doi:10.1016/j.cell.2016.10.017 (2016).

29. Zhou, Y. et al. Metascape provides a biologist-oriented resource for the analysis of systems-level datasets. Nature communications 10, 1523, doi:10.1038/s41467-019-09234-6 (2019).

30. Shannon, P. et al. Cytoscape: a software environment for integrated models of biomolecular interaction networks. Genome research 13, 2498-2504, doi:10.1101/gr.1239303 (2003).

31. Chin, C. H. et al. cytoHubba: identifying hub objects and sub-networks from complex interactome. BMC Syst Biol 8 Suppl 4, S11, doi:10.1186/1752-0509-8-s4-s11 (2014).

32. Luan, H. et al. Identification of Key Prognostic Biomarker and Its Correlation with Immune Infiltrates in Pancreatic Ductal Adenocarcinoma. Disease markers 2020, 8825997, doi:10.1155/2020/8825997 (2020).

33. Yang, X. et al. Study on the Multitarget Mechanism and Key Active Ingredients of Herba Siegesbeckiae and Volatile Oil against Rheumatoid Arthritis Based on Network Pharmacology. Evidence-based complementary and alternative medicine: eCAM 2019, 8957245, doi:10.1155/2019/8957245 (2019).

34. Wu, C. et al. BioGPS: an extensible and customizable portal for querying and organizing gene annotation resources. Genome biology 10, R130, doi:10.1186/gb-2009-10-11-r130 (2009).

35. Loher, P. \& Rigoutsos, I. Interactive exploration of RNA22 microRNA target predictions. Bioinformatics (Oxford, England) 28, 3322-3323, doi:10.1093/bioinformatics/bts615 (2012).

36. Dweep, H., Gretz, N. \& Sticht, C. miRWalk database for miRNA-target interactions. Methods in molecular biology (Clifton, N.J.) 1182, 289-305, doi:10.1007/978-1-4939-1062-5_25 (2014).

37. Tokar, T. et al. mirDIP 4.1-integrative database of human microRNA target predictions. Nucleic acids research 46, D360-d370, doi:10.1093/nar/gkx1144 (2018).

38. Karagkouni, D. et al. DIANA-LncBase v3: indexing experimentally supported miRNA targets on noncoding transcripts. Nucleic acids research 48, D101-d110, doi:10.1093/nar/gkz1036 (2020).

39. Li, J. H., Liu, S., Zhou, H., Qu, L. H. \& Yang, J. H. starBase v2.0: decoding miRNA-ceRNA, miRNAncRNA and protein-RNA interaction networks from large-scale CLIP-Seq data. Nucleic acids research 42, D92-97, doi:10.1093/nar/gkt1248 (2014).

40. Chin, C. H. et al. cytoHubba: identifying hub objects and sub-networks from complex interactome. BMC Syst Biol 8 Suppl 4, S11, doi:10.1186/1752-0509-8-S4-S11 (2014).

41. Wykoff, C. C. et al. Risk of Blindness Among Patients With Diabetes and Newly Diagnosed Diabetic Retinopathy. Diabetes Care 44, 748-756, doi:10.2337/dc20-0413 (2021).

42. Ding, L. et al. Identification of the differential expression of serum microRNA in type 2 diabetes. Bioscience, biotechnology, and biochemistry 80, 461-465, doi:10.1080/09168451.2015.1107460 (2016).

43. Qing, S. et al. Serum miRNA biomarkers serve as a fingerprint for proliferative diabetic retinopathy. Cellular physiology and biochemistry: international journal of experimental cellular physiology, 
biochemistry, and pharmacology 34, 1733-1740, doi:10.1159/000366374 (2014).

44. Yang, Z. et al. miR-7 Reduces High Glucose Induced-damage Via HoxB3 and PI3K/AKT/mTOR Signaling Pathways in Retinal Pigment Epithelial Cells. Current molecular medicine 20, 372-378, doi:10.2174/1566524019666191023151137 (2020).

45. Cao, Y. L., Liu, D. J. \& Zhang, H. G. MiR-7 regulates the PI3K/AKT/VEGF pathway of retinal capillary endothelial cell and retinal pericytes in diabetic rat model through IRS-1 and inhibits cell proliferation. European review for medical and pharmacological sciences 22, 4427-4430, doi:10.26355/eurrev_201807_15493 (2018).

46. Suvas, P., Liu, L., Rao, P., Steinle, J. J. \& Suvas, S. Systemic alterations in leukocyte subsets and the protective role of NKT cells in the mouse model of diabetic retinopathy. Experimental eye research 200, 108203, doi:10.1016/j.exer.2020.108203 (2020).

47. Sonoda, K. H. [Natural Immunity and Ocular Inflammation]. Fukuoka igaku zasshi = Hukuoka acta medica 107, 205-212 (2016).

48. Miyake, S. [MAIT cells in autoimmunity]. Nihon Rinsho Men'eki Gakkai kaishi = Japanese journal of clinical immunology 37, 19-24, doi:10.2177/jsci.37.19 (2014).

49. Czaja, A. J. Incorporating mucosal-associated invariant T cells into the pathogenesis of chronic liver disease. World journal of gastroenterology 27, 3705-3733, doi:10.3748/wjg.v27.i25.3705 (2021).

50. Gazali, A. M. et al. Mucosal-associated invariant T cell alterations during the development of human type 1 diabetes. Diabetologia 63, 2396-2409, doi:10.1007/s00125-020-05257-7 (2020).

51. Chen, M. \& Xu, H. Parainflammation, chronic inflammation, and age-related macular degeneration. Journal of leukocyte biology 98, 713-725, doi:10.1189/jlb.3RI0615-239R (2015).

52. Cherry, J. D., Olschowka, J. A. \& O'Banion, M. K. Neuroinflammation and M2 microglia: the good, the bad, and the inflamed. Journal of neuroinflammation 11, 98, doi:10.1186/1742-2094-11-98 (2014).

53. Zeng, X. X., Ng, Y. K. \& Ling, E. A. Neuronal and microglial response in the retina of streptozotocininduced diabetic rats. Visual neuroscience 17, 463-471, doi:10.1017/s0952523800173122 (2000).

54. Wang, J., Xu, X., Elliott, M. H., Zhu, M. \& Le, Y. Z. Müller cell-derived VEGF is essential for diabetesinduced retinal inflammation and vascular leakage. Diabetes 59, 2297-2305, doi:10.2337/db091420 (2010).

55. Chen, X., Zhou, H., Gong, Y., Wei, S. \& Zhang, M. Early spatiotemporal characterization of microglial activation in the retinas of rats with streptozotocin-induced diabetes. Graefe's archive for clinical and experimental ophthalmology = Albrecht von Graefes Archiv fur klinische und experimentelle Ophthalmologie 253, 519-525, doi:10.1007/s00417-014-2727-y (2015).

56. Butturini, E., Boriero, D., Carcereri de Prati, A. \& Mariotto, S. STAT1 drives M1 microglia activation and neuroinflammation under hypoxia. Archives of biochemistry and biophysics 669, 22-30, doi:10.1016/j.abb.2019.05.011 (2019).

57. Abu El-Asrar, A. M. et al. Interleukin-11 Overexpression and M2 Macrophage Density are Associated with Angiogenic Activity in Proliferative Diabetic Retinopathy. Ocular immunology and inflammation 28, 575-588, doi:10.1080/09273948.2019.1616772 (2020). 
58. Cappellari, R. et al. Diabetes mellitus impairs circulating proangiogenic granulocytes. Diabetologia 63, 1872-1884, doi:10.1007/s00125-020-05142-3 (2020).

59. Majji, A. B. et al. A comparative study of epiretinal membranes associated with Eales' disease: a clinicopathologic evaluation. Eye (London, England) 20, 46-54, doi:10.1038/sj.eye.6701788 (2006).

60. Yoshida, S. et al. Increased expression of M-CSF and IL-13 in vitreous of patients with proliferative diabetic retinopathy: implications for M2 macrophage-involving fibrovascular membrane formation. The British journal of ophthalmology 99, 629-634, doi:10.1136/bjophthalmol-2014-305860 (2015).

61. Ikeda, T., Nakamura, K., Kida, T. \& Oku, H. Possible roles of anti-type II collagen antibody and innate immunity in the development and progression of diabetic retinopathy. Graefe's archive for clinical and experimental ophthalmology = Albrecht von Graefes Archiv fur klinische und experimentelle Ophthalmologie, doi:10.1007/s00417-021-05342-6 (2021).

62. Wang, J. H., Kumar, S. \& Liu, G. S. Bulk Gene Expression Deconvolution Reveals Infiltration of M2 Macrophages in Retinal Neovascularization. Investigative ophthalmology \& visual science 62, 22, doi:10.1167/iovs.62.14.22 (2021).

63. Qi, X. et al. ceRNA in cancer: possible functions and clinical implications. J Med Genet 52, 710-718, doi:10.1136/jmedgenet-2015-103334 (2015).

64. Arutyunov, G. P. et al. [Factors associated with levels of interleukins $-18,-8$, and -6 in hypertensive patients at high and very high cardiovascular risk]. Kardiologiia 57, 69-75, doi:10.18087/cardio.2410 (2017).

65. Sato, T., Takeuchi, M., Karasawa, Y., Takayama, K. \& Enoki, T. Comprehensive expression patterns of inflammatory cytokines in aqueous humor of patients with neovascular age-related macular degeneration. Scientific reports 9, 19447, doi:10.1038/s41598-019-55191-x (2019).

66. Yu, Y. et al. The Profile of Angiogenic Factors in Vitreous Humor of the Patients with Proliferative Diabetic Retinopathy. Current molecular medicine 17, 280-286, doi:10.2174/1566524017666171106111440 (2017).

67. Noma, H. et al. Aqueous Humor Levels of Soluble Vascular Endothelial Growth Factor Receptor and Inflammatory Factors in Diabetic Macular Edema. Ophthalmologica. Journal international d'ophtalmologie. International journal of ophthalmology. Zeitschrift fur Augenheilkunde 238, 81-88, doi:10.1159/000475603 (2017).

68. Yao, Y. et al. Interleukin-6 and Diabetic Retinopathy: A Systematic Review and Meta-Analysis. Current eye research 44, 564-574, doi:10.1080/02713683.2019.1570274 (2019).

69. Jo, D. H. et al. Interaction between microglia and retinal pigment epithelial cells determines the integrity of outer blood-retinal barrier in diabetic retinopathy. Glia 67, 321-331, doi:10.1002/glia.23542 (2019).

70. Wang, Y., Zhai, W. L. \& Yang, Y. W. Association between NDRG2/IL-6/STAT3 signaling pathway and diabetic retinopathy in rats. European review for medical and pharmacological sciences $24,3476-$ 3484, doi:10.26355/eurrev_202004_20806 (2020). 
71. Wang, X., Yu, X., Long, X. \& Pu, Q. MIR205 host gene (MIR205HG) drives osteosarcoma metastasis via regulating the microRNA 2114-3p (miR-2114-3p)/twist family bHLH transcription factor 2 (TWIST2) axis. Bioengineered 12, 1576-1586, doi:10.1080/21655979.2021.1920326 (2021).

72. Singh, A. et al. Differential diagnosis of non-small cell lung carcinoma by circulating microRNA. Journal of cancer research and therapeutics 16, 127-131, doi:10.4103/jcrt.JCRT_872_19 (2020).

73. Degos, C. et al. Omp25-dependent engagement of SLAMF1 by Brucella abortus in dendritic cells limits acute inflammation and favours bacterial persistence in vivo. Cellular microbiology 22 , e13164, doi:10.1111/cmi.13164 (2020).

74. Wang, G. et al. Migration of myeloid cells during inflammation is differentially regulated by the cell surface receptors Slamf1 and Slamf8. PLoS One 10, e0121968, doi:10.1371/journal.pone.0121968 (2015).

75. Babu, H. et al. Systemic Inflammation and the Increased Risk of Inflamm-Aging and Age-Associated Diseases in People Living With HIV on Long Term Suppressive Antiretroviral Therapy. Frontiers in immunology 10, 1965, doi:10.3389/fimmu.2019.01965 (2019).

76. Magnusson, L., Espes, D., Casas, R. \& Carlsson, P. O. Increased Plasma Levels of the Co-stimulatory Proteins CDCP1 and SLAMF1 in Patients With Autoimmune Endocrine Diseases. Frontiers in immunology 11, 1916, doi:10.3389/fimmu.2020.01916 (2020).

77. Tabassum, R. et al. Common variants of SLAMF1 and ITLN1 on 1q21 are associated with type 2 diabetes in Indian population. Journal of human genetics 57, 184-190, doi:10.1038/jhg.2011.150 (2012).

78. Zhong, Z. et al. Circulating microRNA expression profiling and bioinformatics analysis of patients with coronary artery disease by RNA sequencing. Journal of clinical laboratory analysis $\mathbf{3 4}$, e23020, doi:10.1002/jcla.23020 (2020).

79. Sun, H. et al. Expression of exosomal microRNAs during chondrogenic differentiation of human bone mesenchymal stem cells. Journal of cellular biochemistry 120,171-181, doi:10.1002/jcb.27289 (2019).

80. Hadjicharalambous, M. R. et al. Long intergenic non-coding RNAs regulate human lung fibroblast function: Implications for idiopathic pulmonary fibrosis. Scientific reports 9,6020 , doi:10.1038/s41598-019-42292-w (2019).

81. Huang, C. S., Ho, J. Y., Chiang, J. H., Yu, C. P. \& Yu, D. S. Exosome-Derived LINC00960 and LINC02470 Promote the Epithelial-Mesenchymal Transition and Aggressiveness of Bladder Cancer Cells. Cells 9 , doi:10.3390/cells9061419 (2020).

82. Asano, K. et al. Intestinal CD169(+) macrophages initiate mucosal inflammation by secreting CCL8 that recruits inflammatory monocytes. Nature communications 6,7802 , doi: $10.1038 /$ ncomms 8802 (2015).

83. Abu El-Asrar, A. M. et al. The CC chemokines CCL8, CCL13 and CCL20 are local inflammatory biomarkers of HLA-B27-associated uveitis. Acta ophthalmologica 97, e122-e128, doi:10.1111/aos.13835 (2019). 
84. Irak, K., Bayram, M., Cifci, S. \& Sener, G. Serum levels of NLRC4 and MCP-2/CCL8 in patients with active Crohn's disease. PLoS One 16, e0260034, doi:10.1371/journal.pone.0260034 (2021).

85. He, J. et al. Blockade of vascular endothelial growth factor receptor 1 prevents inflammation and vascular leakage in diabetic retinopathy. Journal of ophthalmology 2015, 605946, doi:10.1155/2015/605946 (2015).

86. Capozzi, M. E. et al. The peroxisome proliferator-activated receptor- $\beta / \delta$ antagonist GSK0660 mitigates retinal cell inflammation and leukostasis. Experimental eye research 190, 107885, doi:10.1016/j.exer.2019.107885 (2020).

87. Bai, D. et al. LncRNA CASC15 promotes the proliferation of papillary thyroid carcinoma cells by regulating the miR-7151-5p/WNT7A axis. Pathology, research and practice 225, 153561, doi:10.1016/j.prp.2021.153561 (2021).

88. Shao, J. et al. KCNQ10T1 affects the progression of diabetic retinopathy by regulating miR-1470 and epidermal growth factor receptor. Journal of cellular physiology 234, 17269-17279, doi:10.1002/jcp.28344 (2019).

89. Zhang, Y. et al. Long noncoding RNA KCNQ10T1 induces pyroptosis in diabetic corneal endothelial keratopathy. American journal of physiology. Cell physiology 318, C346-c359, doi:10.1152/ajpcell.00053.2019 (2020).

90. Zhang, C. et al. Long noncoding RNA Kcnq1 ot1 promotes sC5b-9-induced podocyte pyroptosis by inhibiting miR-486a-3p and upregulating NLRP3. American journal of physiology. Cell physiology 320, C355-c364, doi:10.1152/ajpcell.00403.2020 (2021).

\section{Figures}

\section{Figure 1}

\section{Screening of diagnostic markers of human aqueous humor in DR.}

A. A heatmap corresponding to the expression profile of the top 30 DE-miRNAs in human aqueous humor in the GSE140959 dataset as determined based upon P values. Red rectangles represent high expression, and green rectangles represent a low expression.

B. A volcano plot corresponding to the expression profile of miRNAs in aqueous humor in the GSE140959 dataset. The red plots represent upregulated miRNAs, the black plots represent nonsignificant miRNAs, and the green plots represent downregulated miRNAs.

C. The Violin charts show the fold change of the top 3 upregulated miRNAs identified in human aqueous humor.

DE-miRNAs: differentially expressed miRNAs; DR: Diabetic Retinopathy; FC: Fold Change. 


\section{Figure 2}

Detection of differentially expressed mRNAs (DE-mRNAs) of human retina in the GSE160306 dataset.

A. An expression heatmap corresponding to the expression profile of mRNAs in the human retina.

B. A volcano plot corresponding to the expression profile of mRNAs in the human retina. The red plots represent upregulated genes, the black plots represent nonsignificant genes, and the blue plots represent downregulated genes.

DE-mRNAs: differentially expressed mRNAs.

\section{Figure 3}

\section{Evaluation and visualization of immune cell infiltration.}

A. PCA cluster plot of immune cell infiltration between DR samples and control samples.

B. Correlation heat map of 24 types of immune cells. The size of the colored squares represents the strength of the correlation; blue represents a

positive correlation, red represents a negative correlation. The darker the color, the stronger the correlation.

C. Violin diagram of the proportion of 24 types of immune cells. The red marks represent the difference in infiltration between the two group.

\section{Figure 4}

XCell scores of 64 cell types between DR samples and control samples in the GSE160306 dataset.

A-E. Violin charts of lymphoid cells, myeloid cells, stem cells, stromal cells and other cells, respectively.

F. Venn diagram of immune cell intersection of ImmucellAI and xCell results.

\section{Figure 5}


A. The sankey bubble chart shows the top $8 \mathrm{GO}$ enriched terms in $\mathrm{BP}, \mathrm{CC}$ and MF. The most significant $\mathrm{BP}$ involved in cellular defense response, negative regulation of adaptive immune response based on somatic recombination of immune receptors built from immunoglobulin superfamily domains, and negative regulation of adaptive immune response, $\mathrm{CC}$ involved in anchored component of membrane, external side of plasma membrane, and cluster of actin-based cell projections, and MF involved in steroid hydroxylase activity, oxygen binding and chemokine activity.

B. The chord plot shows the most enriched KEGG pathways of DE-mRNAs. The most significant KEGG pathways are involved in phagosome, amoebiasis and salmonella infection.

DE-mRNAs: differentially expressed mRNAs; GO: Gene Ontology; BP: biological process; CC: cellular components; MF: molecular function; KEGG: Kyoto Encyclopedia Genes and Genomes;

The screening criteria for significant enriched biological processes and pathways were $Q<0.05$. The $Q$ value is the adjusted $p$-value.

\section{Figure 6}

\section{PPI network of DE-mRNAs and two cluster modules extracted by MCODE.}

A. The interaction network between proteins coded by DE-mRNAs was comprised of 136 nodes and 65 edges. Each node represents a protein, while each edge represents one protein-protein association. The smaller the value of $Q$ is, the larger the shape size. Six cluster modules were extracted by MCODE.

B. Cluster 1 had the highest cluster score (score: 5, 5 nodes and 10 edges), followed by cluster 2 (C) (score: 3,3 nodes and 3 edges).

\section{Figure 7}

\section{Immune system-specific expressed hub genes identification.}
A. The bar graph shows that the specific expression of DE-mRNA in the haematologic/immune system ranks second $(23 / 91,25.27 \%)$.
B. Cluster plots represent the top 10 hub genes identified by cytoHubba.
C. A Venn diagram of immune system-specific expressed hub genes of the 2 databases.
D. The Violin charts represent the expression of hub gene (IL6, SLAMF1 and CCL8) in the human retina. 


\section{Figure 8}

\section{The construction of ceRNA network of IL6.}
A. A Venn diagram of shared target miRNAs of IL6 of the 3 databases.
B. The bar graph shows that 8 out of 56 shared miRNAs verified by the GSE160308 dataset, including 7 up-regulated and 1 downregulated. The red bar graph means upregulation and the blue means downregulation.
C. The Violin charts represent the expression of hsa-miR-2114-3p identified in retina.
D. The Venn diagram of 36 shared target IncRNAs of hsa-miR-2114-3p of the 2 datasets.
E. Network interaction map of IL6, hsa-miR-2114-3p and 36 shared IncRNAs. Then 3 out 36 shared IncRNA were verified by GSE160306-IncRNA dataset, including 1 up-regulated (PP12613) and 2 down- regulated.
F. The Violin charts represent the expression of PP12613.
G. The ceRNA network was constructed: IncRNA-PP12613/hsa-miR-2114-3p/IL6.

\section{Figure 9}

\section{The construction of ceRNA network of SLAMF1.}

A. A Venn diagram of shared target miRNAs of SLAMF1 of the 3 databases.

B. The bar graph shows that 49 out of 606 shared miRNAs verified by the GSE160308 dataset, including 18 up-regulated and 31 downregulated. The red bar graph means upregulation and the blue means downregulation.

C. The Violin charts represent the expression of hsa-miR-6891-5p identified in retina.

D. The Venn diagram of 120 shared target IncRNAs of hsa-miR-6891-5p of the 2 datasets.

E. Network interaction map of SLAMF1, hsa-miR-6891-5p and 120 shared IncRNAs. Then 14 out 120 shared IncRNA were verified by GSE160306-IncRNA dataset, including 4 up-regulated and 10 downregulated.

F. The Violin charts represent the expression of LINC00960.

G. The ceRNA network was constructed: IncRNA-LINC00960/ hsa-miR-6891-5p/SLAMF1. 


\section{Figure 10}

\section{The construction of ceRNA network of CCL8.}
A. A Venn diagram of shared target miRNAs of CCL8 of the 3 databases.
B. The bar graph shows that 4 out of 67 shared miRNAs verified by the GSE160308 dataset, including 2 up-regulated and 2 downregulated. The red bar graph means upregulation and the blue means downregulation.
C. The Violin charts represent the expression of hsa-miR-7151-3p identified in retina.
D. The Venn diagram of 81 shared target IncRNAs of hsa-miR-7151-3p of the 2 datasets.
E. Network interaction map of CCL8, hsa-miR-7151-3p and 81 shared IncRNAs. Then 3 out 81 shared IncRNA were verified by GSE160306-IncRNA dataset, including 3 down-regulated.
F. The Violin charts represent the expression of KCNQ10T1.
G. The ceRNA network was constructed: IncRNA-KCNQ10T1/hsa-miR-7151-3p/CCL8.

\section{Figure 11}

The technical roadmap of this article and we proposed the novel ceRNA network.

TSEG: Tissue/organ-Specific Expressed Gene

ISEG: Immune Specific Expressed Gene

The red word means upregulation and the green word means downregulation. 